\title{
Systematic motions in the galactic plane found in the Hipparcos catalogue using Herschel's Method
}

\author{
C. Abad $^{1,2}$ and K. Vieira ${ }^{1,3}$
}

\author{
${ }^{1}$ Centro de Investigaciones de Astronomía CIDA, 5101-A Mérida, Venezuela \\ e-mail: abad@cida.ve \\ 2 Grupo de Mecánica Espacial, Depto. de Física Teórica, Universidad de Zaragoza. 50006 Zaragoza, España \\ 3 Department of Astronomy, Yale University, PO Box 208101 New Haven, CT 06520-8101, USA \\ e-mail: vieira@astro.yale.edu
}

Received 1 November 2003 / Accepted 20 July 2005

\section{ABSTRACT}

Two motions in the galactic plane have been detected and characterized, based on the determination of a common systematic component in Hipparcos catalogue proper motions. The procedure is based only on positions, proper motions and parallaxes, plus a special algorithm which is able to reveal systematic trends. Our results come from two stellar samples. Sample 1 has 4566 stars and defines a motion of apex $(l, b)=\left(177^{\circ} 8,3^{\circ} 7\right) \pm\left(1^{\circ} 5,1^{\circ} 0\right)$ and space velocity $V=27 \pm 1 \mathrm{~km} \mathrm{~s}^{-1}$. Sample 2 has 4083 stars and defines a motion of apex $(l, b)=$ $\left(5^{\circ} 4,-0^{\circ} 6\right) \pm\left(1^{\circ} 9,1^{\circ} 1\right)$ and space velocity $V=32 \pm 2 \mathrm{~km} \mathrm{~s}^{-1}$. Both groups are distributed all over the sky and cover a large variety of spectral types, which means that they do not belong to a specific stellar population. Herschel's method is used to define the initial samples of stars and later to compute the common space velocity. The intermediate process is based on the use of a special algorithm to determine systematic components in the proper motions. As an important contribution, this paper sets out a new way to study the kinematics of the solar neighborhood, in the search for streams, associations, clusters and any other space motion shared by a large number of stars, without being restricted by the availability of radial velocities.

Key words. astrometry - Galaxy: open clusters and associations: general - methods: data analysis - stars: kinematics

\section{Introduction}

The proper motion is a linear representation of the real motion of the stars as it is observed by us. It can be defined as the projection of the instantaneous velocity with modulus and direction constant over the celestial sphere. It is inversely proportional to the distance from the star and its certainty depends directly on the accuracy of the individual stellar positions contributing to it. Obviously, most measured proper motions are small because the distance-to-speed ratio is large.

Hipparcos is an astrometric catalogue with accurate positions and excellent proper motions, measured over a short interval of time. It includes positions and proper motions, important information about the stars such as parallax, color and magnitude, but it lacks radial velocities, which are needed to compute space velocities. This situation is regretted by some authors as an important obstacle to study the 3D stellar motions in the solar neighborhood. In this paper we emphasize that when there is a common component of motion in a sample of stars, and if the proper motions are properly analyzed, more information can be obtained about the 3D space motion, in addition to the tangential velocity.
As in a previous paper (Abad et al. 2003, from now on (A03)), we present a treatment of the proper motions based on Herschel's Method (Trumpler \& Weaver 1953) and the Polinomio Deslizante (Stock \& Abad 1988). Herschel's method reveals the existence of an apex when a common motion is present. A simple equation relates proper motions and the space velocity of the group, through the angular distance from the apex to the stars' position on the celestial sphere, when parallaxes are known. The Polinomio Deslizante (from now on, PD) is a numerical routine that is able to detect systematic trends in a sample of data, which, in our particular case are proper motions. Most previous work on the local kinematics using proper motions (and radial velocities in some cases) usually employed a statistical analysis and adopted an analytical model for the velocity distribution to which proper motions are fitted, through a given set of parameters. The Polinomio Deslizante has no assumptions about the data or their distribution, so its results represent more faithfully the observed reality, not being forced to follow a given equation. A separate mention must be made of Dehnen (1998), who uses a non-parametric maximum penalized likelihood method to estimate the velocity distribution $f(v)$ of nearby stars, based on the positions and tangential 
velocities of a kinematically unbiased sample of 14369 stars selected from Hipparcos catalogue. He found that $f(v)$ shows a rich structure in the radial and azimuthal motions, but not in the vertical velocity. Some of these structures are related to well-known moving groups.

Herschel's original idea was to use the Convergent Point Method to determine the solar motion. Later it was used as an additional tool to confirm the membership of stars in clusters and associations. In this paper we show that this method has more useful features. The substitution of great circles, which represent the stellar proper motions, by their corresponding poles, allows the matching of both position and proper motion with a point on the celestial sphere, for each star. In the case of a stellar cluster, the poles of the cluster members are located on arcs of a great circle over the celestial sphere, but if the apex is located inside the cluster or the stars members are spatially distributed around the Sun, then the poles span along a whole great circle. Using the poles it is possible to extend Herschel's method from small fields to large and dense catalogues, representing the proper motions in a more interesting way than the classical Vector-Point Diagram, because the field of representation is now the whole celestial sphere. The denser the stellar field is, the more crowded the poles are, but this representation highlights in a visual way, important characteristics of the common motion, if there is any in the sample studied.

Stellar associations, clusters, streams and any kinematically bound group in general, can give us some information about the local (and even the global) dynamical structure of the Galaxy. The main interest of this paper is to demonstrate a procedure to detect and compute the motion of any of these possible types of common stellar motions, by looking for systematic trends in the proper motions of many stars, covering large areas on the sky. Using this method on the Hipparcos catalogue, we have found two large-scale patterns of motion, both of them constrained to the galactic plane. One motion is directed radially inwards to the galactic center, while the other is directed radially outwards. For each of them, the apex and velocity are measured.

As Hipparcos stars are near the Sun, it can be difficult to associate these and others similar "streams" to a specific origin. A list of well studied dynamical perturbations that could affect the solar neighborhood, possibly linked to these patterns of motions, includes for example, the Outer Lindblad Resonance (OLR) located just inside the solar radius and produced by the galactic bar, according to Mulbahuer \& Dehnen (2003). Another example, is the ongoing disintegration of the Sagitarius dwarf galaxy, which is crossing the galactic plane as it is being accreted by the Milky Way, at a distance of only one $\mathrm{kpc}$ from the Sun (Majewski et al. 2003). The proximity of the Sagitarius galactic arm can be listed as well. Their effects on the local galactic kinematics are not quantitatively well known, although some qualitative insights can be determined.

\section{The polar representacion of the convergent point method (CPM)}

The great circle defined for each star, by its position and proper motion vector, has been used as a representation of the proper motion in several investigations. For example
Schwan (1991), selected stars of the Hyades open cluster and computed its apex based on the great circles and their intersections. Both, Agekyan \& Popovich (1993) and Jaschek \& Valbousquet (1992), used the CPM to determine the solar motion from different catalogues and numbers of stars. More recently Chereul et al. (1998) determined the space velocity distribution of A-F stars in the Hipparcos data.

In Abad (1996), the intersections between great circles was used as a tracer of the existence of stellar clusters. The study of the density distribution of the intersections helped him to get the apex of the Coma Berenices open cluster. A more mathematical treatment on a global proper motion convergence map has been made by Makarov et al. (2000), working on a sample of X-ray stars from the ROSAT All-Sky Survey Bright catalogue. We show in this paper that the CPM, through the polar representation of the great circle (see (A03)), has more benefits to offer.

Each great circle is uniquely defined by its pole $\boldsymbol{p}$, computed by the cross product of the initial $\boldsymbol{x}_{\boldsymbol{i}}$ and final position $\boldsymbol{x}_{\boldsymbol{f}}$ of the star, using the proper motion according to the following equations:

$$
\begin{aligned}
\boldsymbol{x}_{\boldsymbol{i}}= & (\cos l \cos b, \sin l \cos b, \sin b) \\
\boldsymbol{x}_{\boldsymbol{f}}= & \left(\cos \left(l+\mu_{l} \Delta t\right) \cos \left(b+\mu_{b} \Delta t\right),\right. \\
& \left.\quad \sin \left(l+\mu_{l} \Delta t\right) \cos \left(b+\mu_{b} \Delta t\right), \sin \left(b+\mu_{b} \Delta t\right)\right) \\
\boldsymbol{p}= & \boldsymbol{x}_{\boldsymbol{i}} \times \boldsymbol{x}_{\boldsymbol{f}}
\end{aligned}
$$

with $\Delta t=1$ year. Figure 1 gives a comparative view of both, the CPM and the Vector-Point Diagram, when applied to the Hipparcos catalogue. To illustrate the CPM, we use the polar representation. This figure shows the existence of an area on the celestial sphere with a major concentration of poles. Given that it is possible to fit a great circle to the data, the interpretation of this plot may be related to the existence of a systematic component of the stellar proper motions in the Hipparcos catalogue. In all plots of the whole celestial sphere in this paper, galactic longitude runs from $l=0^{\circ}$ to $l=360^{\circ}$ in order to make it easier to visualize the results.

\section{Solar motion and galactic rotation}

Two well known sources of systematic motion, solar motion and differential galactic rotation, are shared by all the stars around the Sun.

The shape of the feature observed in Fig. 1, made us think that it was a reflex of the solar motion. Actually, this idea was already studied in (A03), using the CPM+PD as we propose here. In their paper, solar motion is obtained as the common trend of motion shared by all stars in Hipparcos Catalogue. As their method requires parallaxes, only stars with $3 \sigma_{\pi} \leq \pi$ were selected, to avoid further problems. Their results are $\left(l_{\odot}, b_{\odot}\right)=$ $\left(61^{\circ} 37,19^{\circ} 95\right) \pm\left(3^{\circ} 13,2^{\circ} 62\right)$ and $V_{\odot}=21.96 \pm 3.72 \mathrm{~km} \mathrm{~s}^{-1}$. It is well known from all papers about solar motion, that the results depend on the stellar sample used. For example, considering stars of only a given spectral type, and comparing results between different samples, reveals what is known as the "asymmetric drift". As the true solar motion is not 


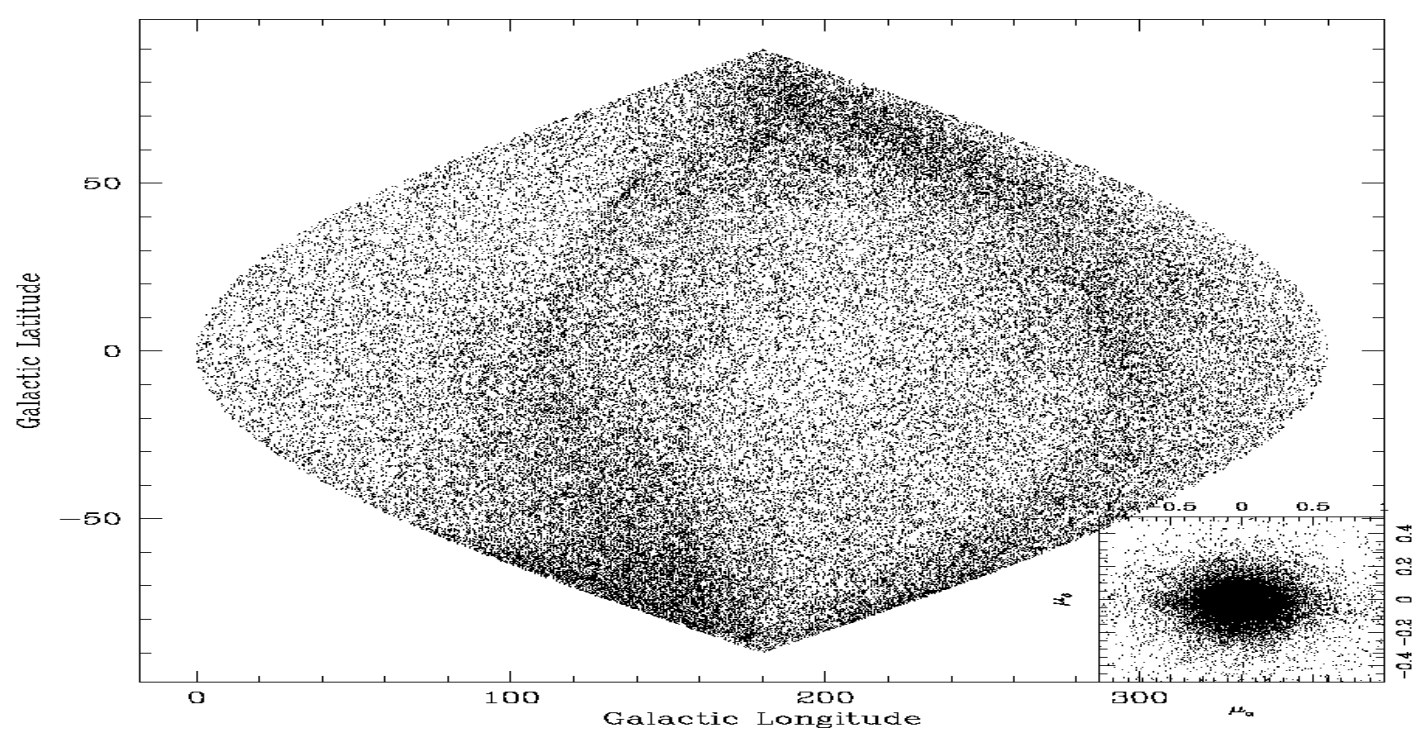

Fig. 1. A comparative view of the Convergent Point Method (CPM) and the Vector-Point Diagram, applied to Hipparcos catalogue. CPM is plotted using the poles of the great circles. An area of enhanced density of poles stands out, on which is possible to fit a great circle.

responsible for this, then these results are basically biased by how the samples were defined.

Any method to find the solar motion, rests on the ability to find a stellar sample with no significant kinematic deviation with respect to the to the Local Standard of Rest (LSR). Particularly in (A03), the solar motion was obtained based on what the PD detected in the whole sample of Hipparcos stars, regardless of spectral type and only limited by having good parallaxes. Precisely the fact that solar motion is present in all stellar proper motions, regardless of any condition, justifies such a choice.

After correcting for both, galactic rotation and solar motion, we are then in a reference frame where all stars have zero common motion. There is no guarantee at all that this "zero common motion reference frame" has no net motion with respect to the LSR, but this is in our opinion the closest realization we have of the LSR, based exclusively in data and with no theoretical assumptions.

Using (A03) values of $\left(l_{\odot}, b_{\odot}\right)$ and $V_{\odot}$, the effect of the solar motion in the stellar proper motion, $\left(\mu_{l, \odot}, \mu_{b, \odot}\right)$ for a star with position $(l, b)$ is given by:

$\mu_{l, \odot} \cos b=\frac{\pi}{4.74}\left(u_{\odot} \sin l-v_{\odot} \cos l\right)$

$\mu_{b, \odot}=\frac{\pi}{4.74}\left(u_{\odot} \cos l \sin b+v_{\odot} \sin l \cos b-w_{\odot} \cos b\right)$

$\left(u_{\odot}, v_{\odot}, w_{\odot}\right)=V_{\odot}\left(\cos l_{\odot} \cos b_{\odot}, \sin l_{\odot} \cos b_{\odot}, \sin b_{\odot}\right)$

where $\left(u_{\odot}, v_{\odot}, w_{\odot}\right)$ are the rectangular components of the solar motion in $\mathrm{km} \mathrm{s}^{-1}$, directed towards the galactic center, the direction of galactic rotation and the galactic north pole, respectively. $\pi$ is the parallax of the star.

Differential galactic rotation also produces part of the observed proper motions, introducing a systematic component which depends mostly on the position in the galaxy, but it is independent of the distance to the star. Oort's constants $A$ and $B$ are used to make simple models of the differential galactic rotation, although more sophisticated methods
(Mignard 2000) express the galactic rotation in terms of the Generalized Oort's constants. We particularly consider Eqs. (27) and (28) of Mignard (2000), to model the differential galactic rotation:

$$
\begin{gathered}
\mu_{l, g r} \cos b=\bar{A} \cos b \cos (2 l-2 \phi)+A^{\prime} \sin b \cos (l-\psi) \\
+B \cos b-B^{\prime} \sin b \cos (l-\chi) \\
\mu_{b, g r}=-\frac{\bar{A}}{2} \sin 2 b \sin (2 l-2 \phi)+A^{\prime} \cos 2 b \sin (l-\phi) \\
-\frac{K}{2} \sin 2 b+B^{\prime} \sin (l-\chi)
\end{gathered}
$$

where the values of the Generalized Oort's constants $\bar{A}$, $A^{\prime}, B, B^{\prime}$ and $K$, which depend on the spectral type, are listed in Table 5 of Mignard (2000).

Both $\left(\mu_{l, \odot}, \mu_{b, \odot}\right)$ and $\left(\mu_{l, g r}, \mu_{b, g r}\right)$ are subtracted from the individual stellar proper motions. Figure 2 shows the polar representation of the proper motions after they are corrected for these two effects. This plot represents the intrinsic motions of the stars, referred to the LSR. Once again, it is still possible to see the dense area of poles making a wide band surrounding the celestial sphere, but with a small change of orientation when compared to Fig. 1. This tells us two new things: (a) solar motion was not the origin, and (b) it is not a common systematic trend for the totality of the stars in the catalogue, but for a subsample. It is necessary to stress that the differential galactic rotation model of Mignard (2000), does not produce the great circle of poles like the one found in Fig. 2.

\section{Selecting stars with common space motion}

Figure 2 shows clearly a band with an enhanced density of poles. The possibility of fitting a great circle would imply the existence of a common space motion. The width of the band represents the internal dispersion around the mean motion of the group. Of course, proper motion errors also play a role and another factor could be a possible selection effect of the 


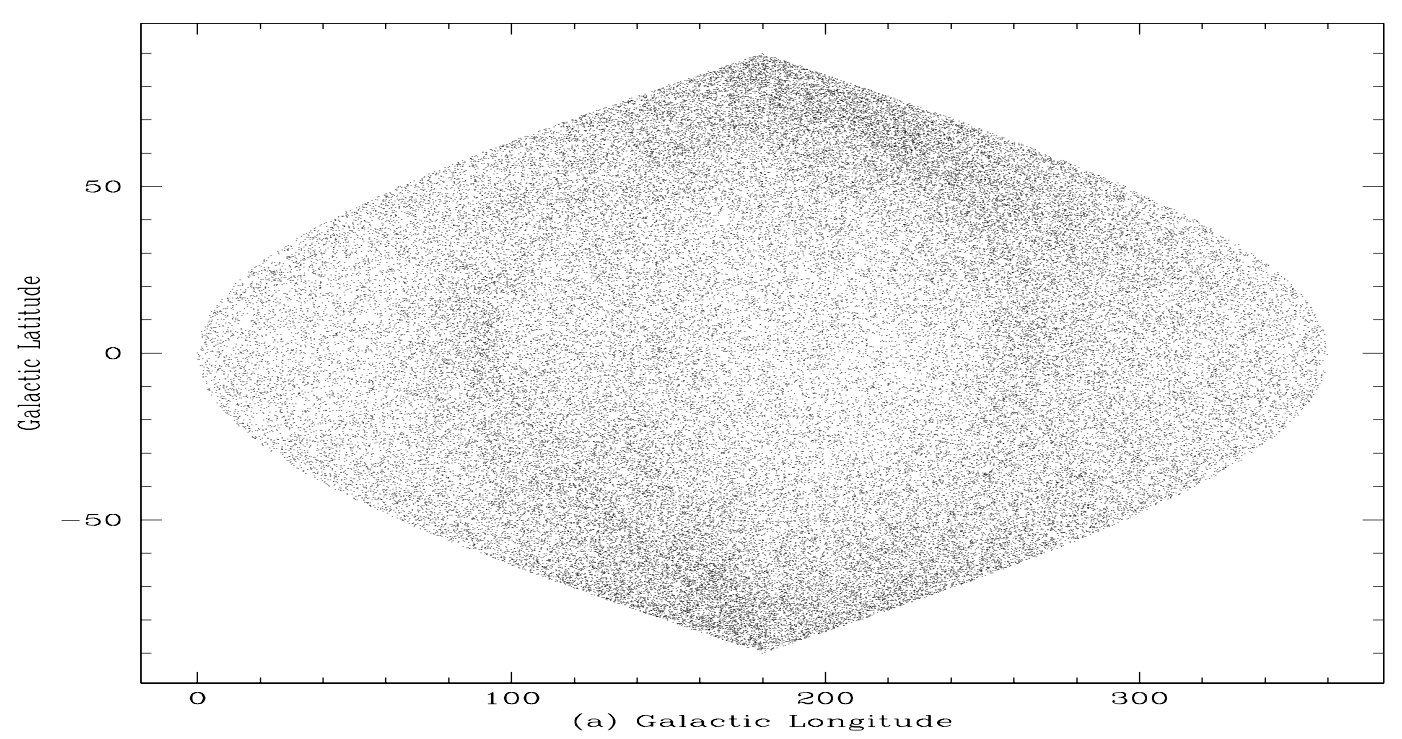

Fig. 2. Polar representation of the CPM for the same stars as Fig. 1, once proper motions have been corrected for solar motion and differential galactic rotation. Therefore, these proper motions are referred to the Local Standard of Rest (LSR). When compared to Fig. 1, it is still possible to see the dense area of poles but with a small change of orientation.

Hipparcos mission, although we later prove that this band corresponds to a real space motion.

Not all of the stars are physical members of this wide great circle, therefore the first step is to select the stars on it and search for possible members to get the modulus and direction of their motion. A half-width of $14^{\circ}$ was chosen around the best fitting central great circle. If we think of this great circle as an "equator", then the corresponding "poles" $\left(\left(l_{0}, b_{0}\right)\right.$ and its opposite) are probable apexes of the motion. Then, it is necessary to separate the stars producing the band into two samples, depending on the direction of their proper motions: those pointing towards $\left(l_{0}, b_{0}\right)$, hereafter Sample 1, and those pointing the other way, hereafter Sample 2. Then, making use of parallaxes, the corrected proper motions are re-scaled to a fixed distance of $100 \mathrm{pc}$ and we apply the PD fitting function on them, in a similar way to that done (A03). If there is a common space motion in these stars, the re-scaled proper motions $\mu_{\text {scaled }}$ have a common behavior, that can be detected and measured by the PD.

For any given point on the sky, the PD produces a vector which represents the common component, shared by all the stars surrounding the point up to a given predefined radius. Therefore two points separated by twice this radius have output vectors completely independent, given that the proper motion data contributing to their computation are distinct. To easily visualize global and/or local patterns in the PD resulting vectors, we define an evenly distributed grid of points all over the sky. Working on Sample 1 for example, after a first application of the PD, a global vectorial pattern of proper motions is found. To verify if it corresponds to a common space motion, all following conditions have to be fulfilled: (a) the pattern must be smooth and systematic all over the sky, (b) both apex and antapex must exist, and (c) the modulus of the vectors obtained with the PD must roughly follow Hershel's formulation, meaning that the modulus of the vectors depend on the angular distance to the apex, being maximum at 90 degrees from it and close to zero in both apex and antapex.
To proceed later with Herschel's method, we define another grid of points, this time making meridians and parallels as if $\left(l_{0}, b_{0}\right)$ and its opposite were the poles of the sphere. The PD is applied on these points and conditions (a), (b) and (c) are checked again. Results of this procedure are shown in Fig. 3 for Sample 1. A clear global pattern is observed. Zooming in on the area of minimum-modulus vectors (Fig. 4c) helps us to select an apex. Additionally, the CPM poles of the PD vectors, fall in a quite narrow great circle that is fitted and from this we measure a better apex.

For each meridian of the grid defined by the apex, we plot the PD vectors modulus $\mu_{\mathrm{PD}}$ versus their position along the meridian (equivalent to the angular distance $\lambda$ from the vector position to the apex). Actually, we transform those moduli to their corresponding tangential velocity in $\mathrm{km} \mathrm{s}^{-1}$, using $V_{\mathrm{t}}=4.74 \mu_{\mathrm{PD}} 100.0$ given that all proper motions (in "/yr) were rescaled to a distance of $100 \mathrm{pc}$. Figure 4a plots the corresponding value of $V_{\mathrm{t}}$ for all vectors of Fig. 3, distributed by meridians. According to Herschel's formulation, for a perfect common space motion, each meridian satisfies $V_{\mathrm{t}}=V \sin (\lambda)$, where $V$ is the total space velocity of the motion, and those vectors located at $\lambda=90^{\circ}$ have $V_{\mathrm{t}}=V$. Figure $4 \mathrm{a}$, can certainly be fitted by a sine function.

Simultaneously, the modulus of the vectors should remain constant for each parallel of the grid. The constant modulus of the vectors located on the equator of the grid, should have the maximum value, because all of them are $90^{\circ}$ from the apex, and their corresponding $V_{\mathrm{t}}$ matches the space velocity $V$ of the motion. Figure $4 \mathrm{~b}$ shows $V_{\mathrm{t}}$ along the different parallels in the grid, for the same proper motion vectors as in Fig. 3.

Finally, based on the $V$ value obtained from the sine function fitted in Fig. 4a, we make a histogram of the ratio $R=$ $\left(4.74 \mu_{\text {scaled }} 100.0\right) /(V \sin (\lambda))$ for each star, as shown in Fig. $4 d$. The true $V$ of the motion should produce a peak in the histogram around $R=1$, though a slightly different value will just shift the position of the peak. More important is the fact 


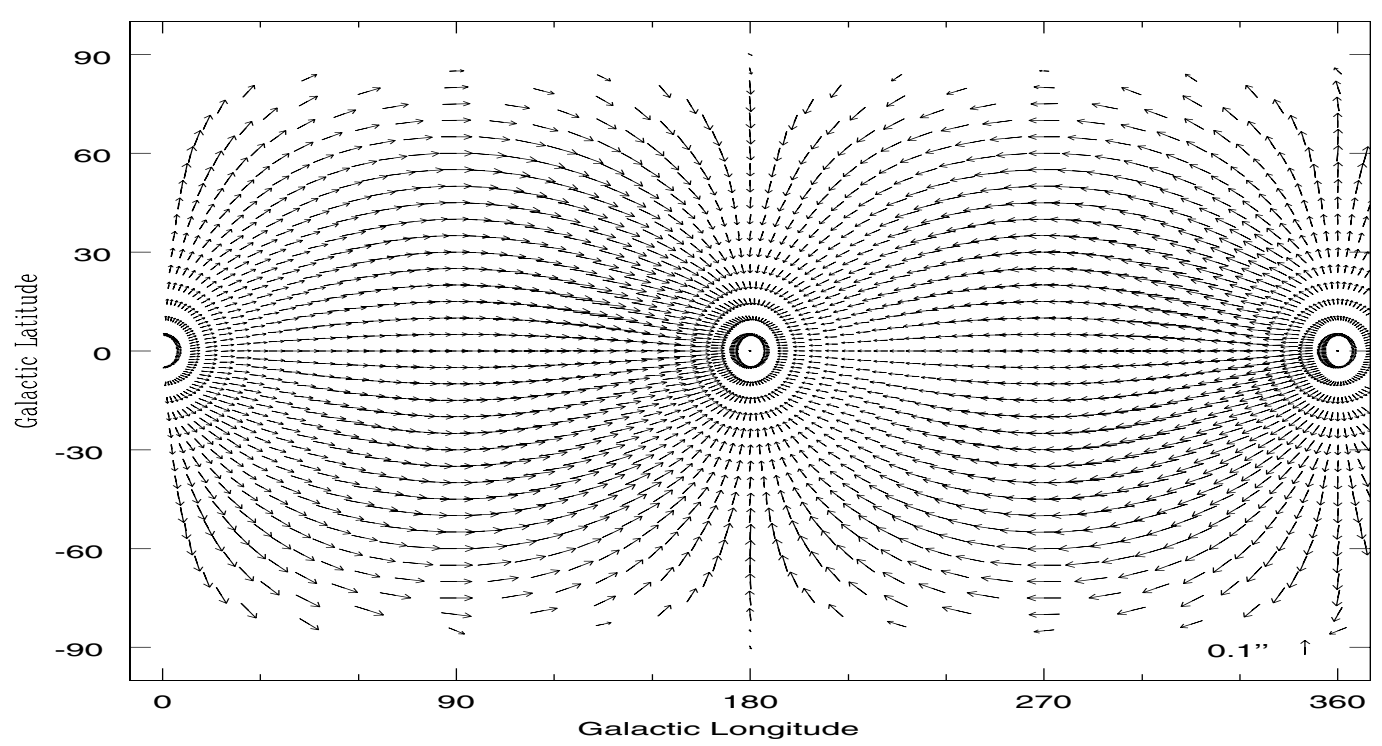

Fig. 3. Vectors of proper motions produced by the Polinomio Deslizante, applied to those stars whose paths are within a $14^{\circ}$ radius centered on $(l, b)=(180,0)$ and taking this point as the apex. This was the first pre-selection of stars done for Sample 1.
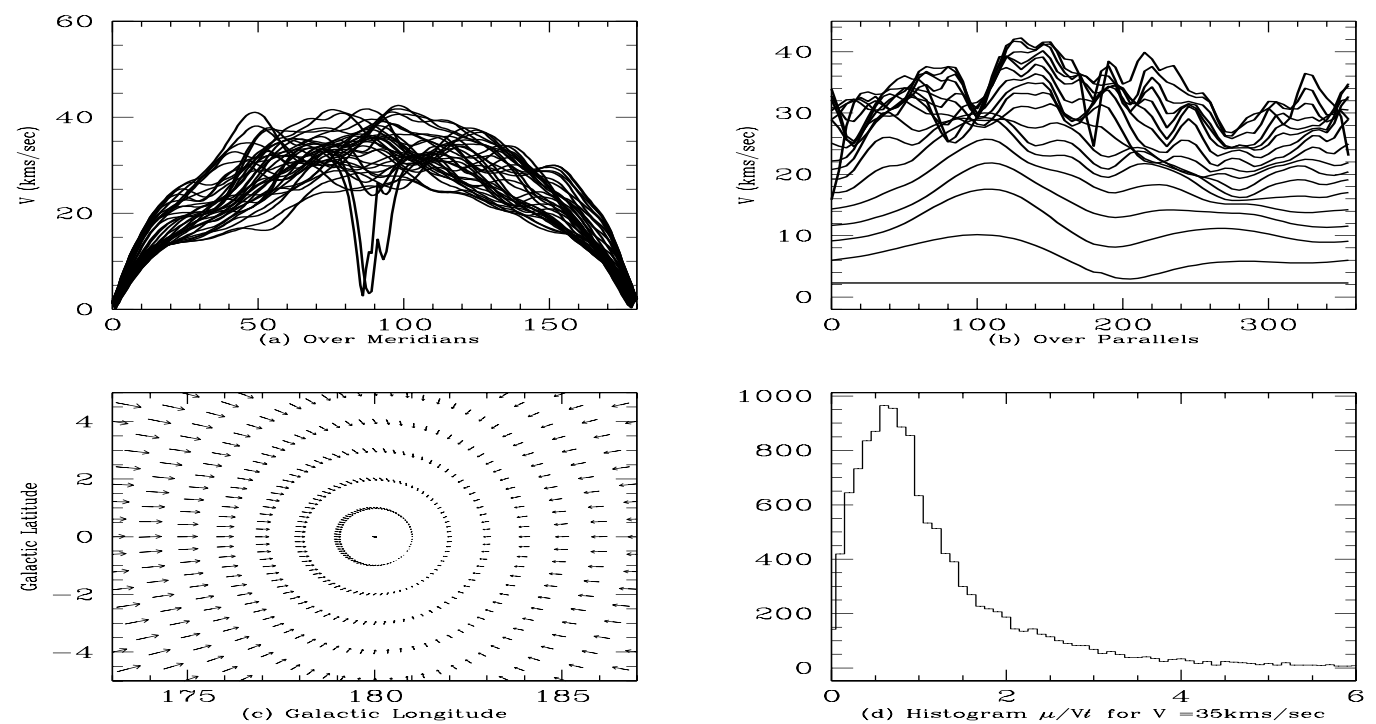

Fig. 4. a) $V_{\mathrm{t}}$ along meridians are similar and can be fit by a sine function of the angular distance $\lambda$ to the apex. b) $V_{\mathrm{t}}$ along parallels. c) An apex point with a positive increment on longitude and negative increment on latitude is closer to the true apex. d) The histogram indicates that the real total space velocity is lower than the initially chosen. Irregular values around $\lambda=90^{\circ}$ come from trigonometric singularities for proper motion vectors located close to the galactic poles $b= \pm 90^{\circ}$.

that clear outliers can be easily rejected, for example in Fig. 4d those stars with $R>4$ were eliminated. In addition to this "comparison of length" we also check the angle between the observed $\mu$ and the kinematics proper motion $\mu_{\text {kin }}$ the star should have, if it were perfectly directed towards the apex with an exact velocity of $V$. Those stars with very large angles were rejected. The purpose of this cleaning is just to get rid of outliers. A new apex is then calculated and the process is iterated.

\section{Two preferential patterns of motion}

The first preselection of stars, done as explained at the beginning of Sect. 4, consisted of 24486 stars from Hipparcos catalogue, 13208 with proper motion towards $(l, b)=(180,0)$, named Sample 1, and 11278 with proper motion towards $(l, b)=(0,0)$, named Sample 2. Stars with $3 \sigma_{\pi}>\pi$ and Hyades cluster members had previously been rejected. We will see later that our final results do not change, even including the Hyades cluster (which has a strong and well defined motion).

\subsection{Sample 1}

Figure 5 shows the final results for Sample 1, after a few iterations of the procedure explained in Sect. 4. The number of selected stars is now reduced to 4566 , and they produce a coherent systematic pattern of motion with apex $(l, b)=$ $\left(177^{\circ} 8,3^{\circ} 7\right) \pm\left(1^{\circ} 5,1^{\circ} 0\right)$ and velocity $V=27.2 \pm 1.1 \mathrm{~km} \mathrm{~s}^{-1}$. Sample 1 stars are located all around the sky and do not 

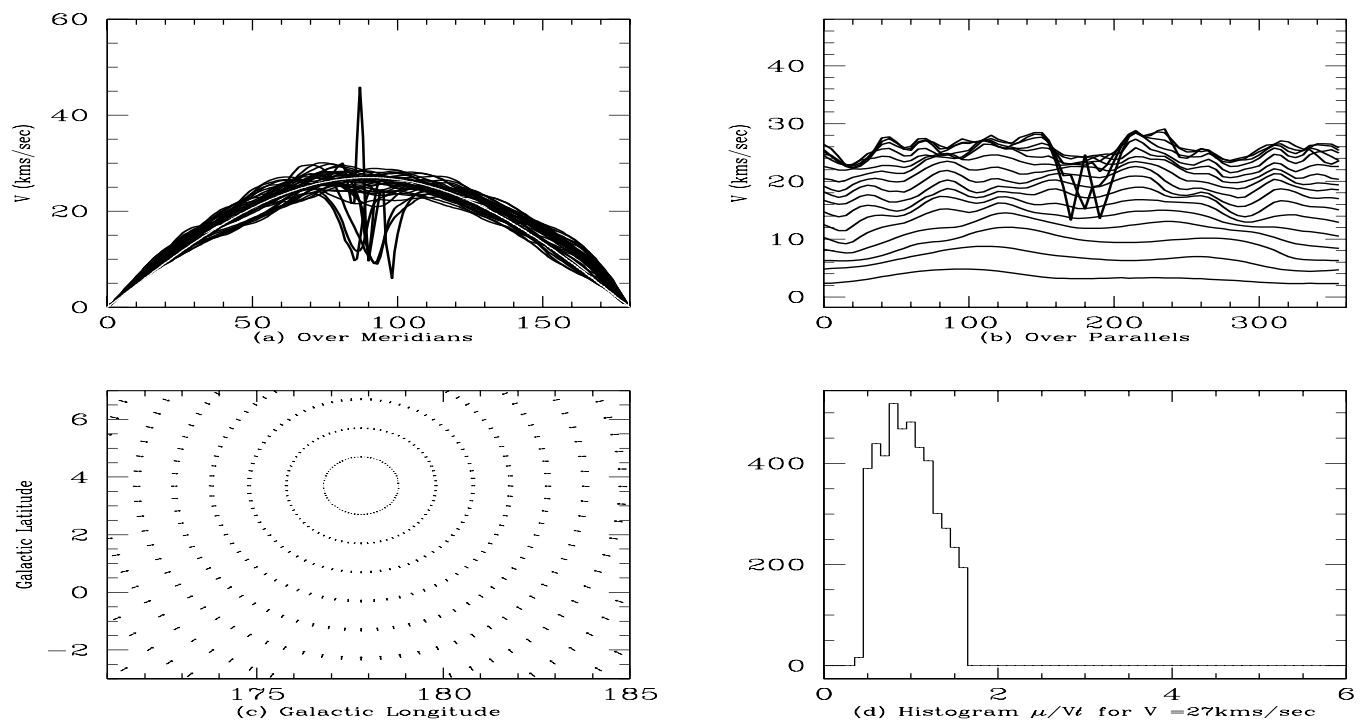

Fig. 5. Final results for Sample 1. The motion is directed to $(l, b)=\left(177^{\circ} 8,3^{\circ} 7\right)$ with a space velocity of $V=27.2 \mathrm{~km} \mathrm{~s}^{-1}$. Irregular values around $\lambda=90^{\circ}$ come from trigonometric singularities for PD vectors located close to the galactic poles $b= \pm 90^{\circ}$.
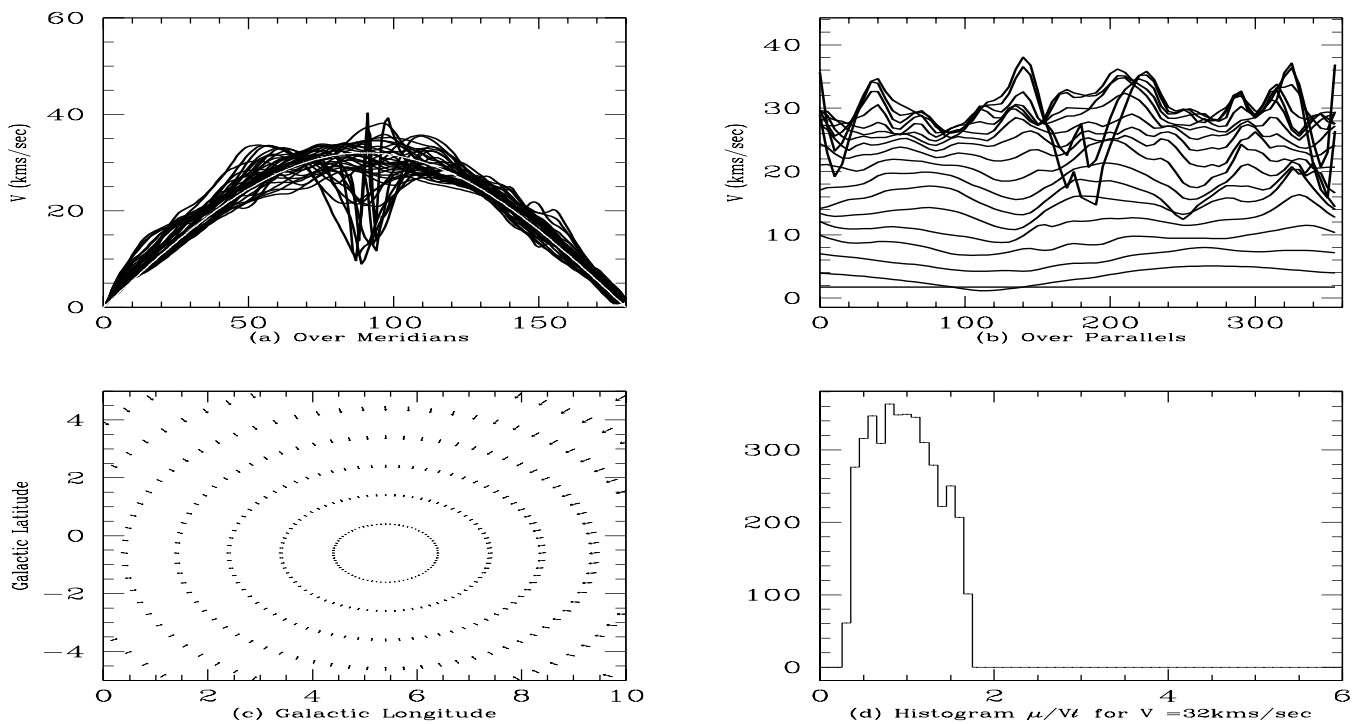

Fig. 6. Final results for Sample 2. The motion is directed to $(l, b)=\left(5^{\circ} 4,-0^{\circ} 6\right)$ with a space velocity of $V=32.1 \mathrm{~km} \mathrm{~s}^{-1}$. Irregular values around $\lambda=90^{\circ}$ come from trigonometric singularities for PD vectors located close to the galactic poles $b= \pm 90^{\circ}$.

exhibit any particular preferred condition on position, distance (space distribution $(X, Y, Z)$, Fig. 8), color, magnitude or spectral type (color-magnitude diagram, Fig. 7). These stars do not display any noticeable difference with the general distribution of Hipparcos stars.

\subsection{Sample 2}

Figure 6 shows the final results for Sample 2. The number of selected stars is now reduced to 4083, and they produce a coherent systematic pattern of motion with apex $(l, b)=$ $\left(5^{\circ} 4,-0^{\circ} 6\right) \pm\left(1^{\circ} 9,1^{\circ} 1\right)$ and velocity $V=32.1 \pm 2.1 \mathrm{~km} \mathrm{~s}^{-1}$. Sample 2 stars, like those of Sample 1, do not exhibit any particular preferred condition (Fig. 7), except for a $45^{\circ}$ tilted preferential line on the $(X, Y)$ plane and a slightly tilted orientation in the $(Y, Z)$ plane (Fig. 8).

\section{Cross-checking the results}

The space motion of a star is the vector sum of its tangential and radial components. If available, radial velocities can be used as an independent test of the results obtained using only proper motions. This is true, given that for a common space motion $V_{r}=V \cos (\lambda)$ so that radial velocities complement tangential velocities for each star.

With this purpose in mind, we use the "Catalogue Général de Vitesses Radiales Moyennes pour les Etoiles Galactiques" (Barbier-Brossat \& Figon 2000). The catalogue contains mean radial velocities for 36145 stars of which 1198 were matched 


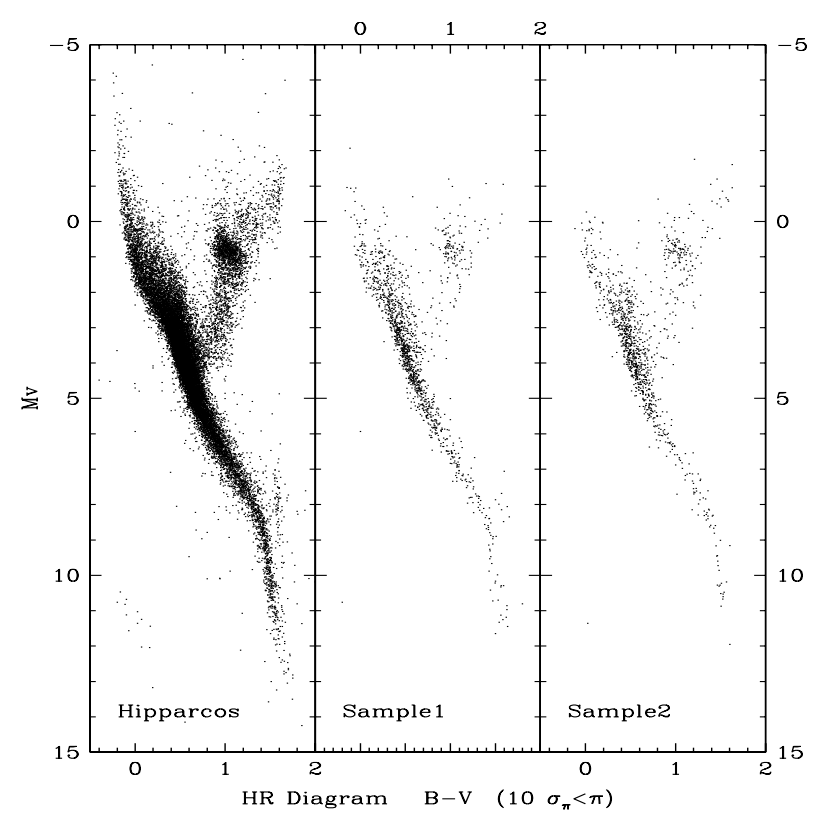

Fig. 7. Color-Magnitude Diagram for Hipparcos catalogue and Samples 1 and 2, including only stars with $3 \sigma_{\pi}<\pi$. Distributions are similar.

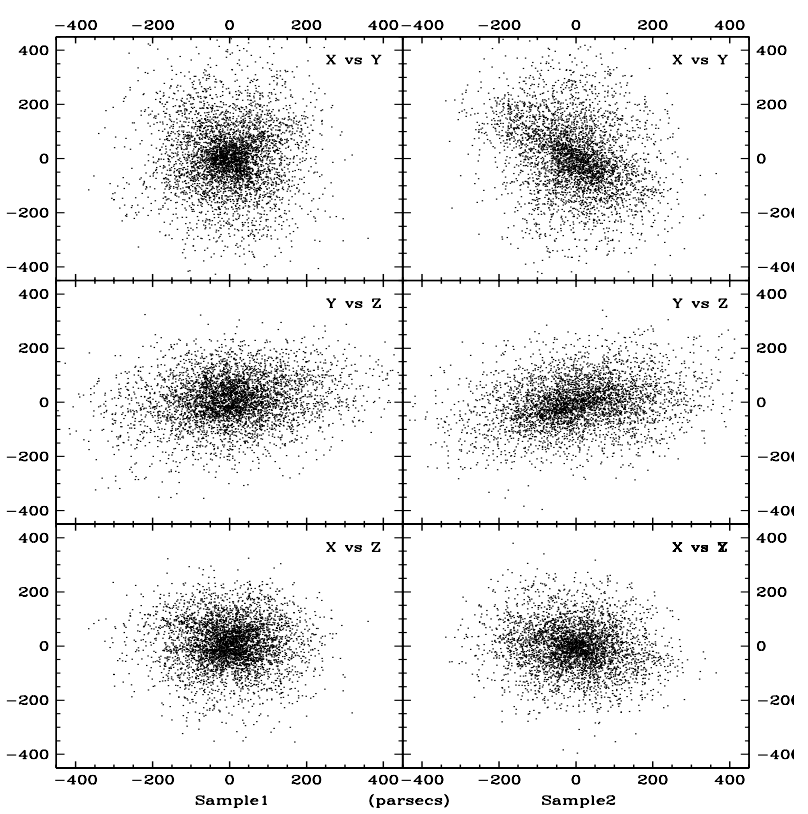

Fig. 8. Spatial distribution $(X, Y, Z)$ for Sample 1 and 2 . There is a $45^{\circ}$ tilted $(X, Y)$ preferential line and a $(Y, Z)$ slightly tilted orientation for stars of Sample 2.

with final Sample 1 and 886 with final Sample 2. Figure 9 plots for each sample, radial velocity versus galactic longitude, once corrected for solar motion.

The existence of a tendency in the radial velocities, which properly complements the patterns found in the proper motions, is evident for Sample 1. Extreme and opposite values around $\pm 27 \mathrm{~km} \mathrm{~s}^{-1}$, are found close to $l=0^{\circ}$ and $l=180^{\circ}$. Minimum values of $0 \mathrm{~km} \mathrm{~s}^{-1}$ are observed around $l=90^{\circ}$ and $l=270^{\circ}$. A similar, but noisier trend is observed for Sample 2.
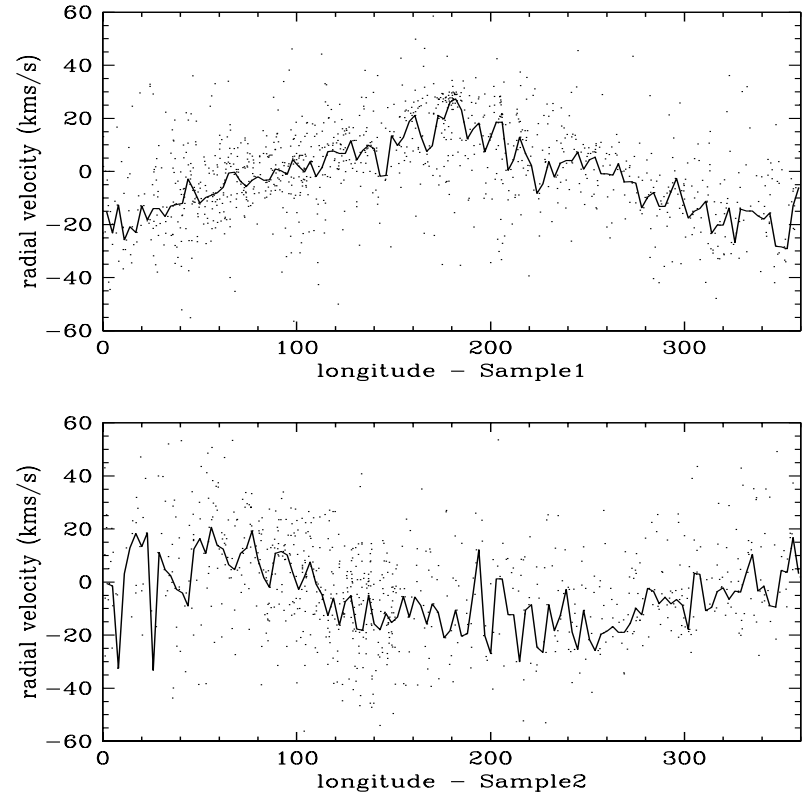

Fig. 9. Distribution of radial velocities vs. galactic longitude for those stars of the "Catalogue Général de Vitesses Radiales Moyennes pour les etoiles galactiques" included into Samples 1 and 2. 1198 stars belong to Sample 1 (upper figure) and 886 stars to Sample 2 (lower figure). In both cases the radial velocities have been corrected for solar motion. Median values over $4^{\circ}$ longitude interval are plotted with a solid line. Excellent agreement with the results shown in Fig. 5 exists for Sample 1.

\section{Cross-checking the method}

With the only aim of checking the correctness and robustness of the method, we apply the PD+CPM poles to several "dynamical streams" detected by Famaey et al. (2005) (from now on (F05)). In their paper, a maximum-likelihood method based on a Bayesian approach is used to derive the kinematic properties of several subgroups. A sample of around 6000 giant stars, with proper motion, parallax and radial velocity data (Tycho-2, Hipparcos and CORAVEL), are used to reconstruct 3D space velocities of the stars.

Figure 11 shows the polar representation of the different stellar streams and groups as listed by (F05). the Young Giants, the Hyades-Pleiades supercluster, the Hercules stream, the Sirius moving group and the so-called Smooth Background. The proper motions from which these poles were computed, are already corrected for differential galactic rotation and solar motion, using the values and equations cited in (F05), as explained in next parapraph. These corrections assure us that whatever the PD detects later as a common motion in any of these samples, corresponds to a real intrinsic spatial motion and not to a common component induced by some external effect. We first use (F05) values to be consistent with their work, although we later try (A03) values as well.

Following (F05), given the observed proper motions $\mu_{l, 0}$ and $\mu_{b, 0}$, the corrected values for galactic rotation are:

$$
\begin{aligned}
\kappa \mu_{l} & =\kappa \mu_{l, 0}-A \cos 2 l-B \\
\kappa \mu_{b} & =\kappa \mu_{b, 0}+1 / 2 A \sin 2 b \sin 2 l
\end{aligned}
$$


where $\kappa=4.74$ is the factor to convert proper motions into space velocities. $A=14.82 \mathrm{~km} / \mathrm{s} / \mathrm{kpc}$ and $B=-12.37 \mathrm{~km} / \mathrm{s} / \mathrm{kpc}$ are the Oort constants, derived by Feast \& Whitelock (1997). The effect of solar motion on the proper motion of the stars is computed following Eqs. (4) and (5), with $\left(u_{\odot}, w_{\odot}\right)=$ $(10.24,7.77) \mathrm{km} \mathrm{s}^{-1}$, as measured by (F05). Knowing that velocities along the direction of galactic rotation are affected by the asymmetric drift, (F05) adopted the value of $v_{\odot}=$ $5.25 \mathrm{~km} \mathrm{~s}^{-1}$ from Dehnen \& Binney (1998).

It is straightforward to see that the stars in the Young Giants group, do not exhibit a particular trend in their poles, except some accumulation near the galactic poles. This is consistent with these stars being young disk population. Similarly, the High-Velocity stars (not shown in Fig. 11), have their poles more or less randomly distributed across the celestial sphere.

On the other hand, the Hyades-Pleiades supercluster's poles are located on a band, close to the wide great circle of poles detected by us (see Fig. 2). Even more, our pattern is also visible in the poles of the stars of the Smooth Background, which according to (F05), corresponds to an "axisymmetric" mixed population of the galactic disk, composed of stars born at many different epochs.

The Sirius and Hercules streams deserve special attention, since each have their poles located along and around the arc of a great circle, clearly biased because (F05) only use stars from the northern hemisphere. The lower right panel of Fig. 11 shows that both distributions may be complementary. Based on these distributions it is clear that both motions are almost opposed and close to the galactic plane. We most stress here that we are not relating Sirius and Hercules streams to the pattern of motions we found in Hipparcos. What is really interesting for us is that the distribution of their poles, as seen in Fig. 11 right lower panel, also lies on a great circle. This offers us the opportunity to test our method and verify that it is able to detect, separate and measure their corresponding apex and velocity.

After correcting for galactic rotation and solar motion, the proper motions are re-scaled to a distance of $100 \mathrm{pc}$ and the PD is applied. For this, a grid of points separated by 10 degrees is defined and we set a $15^{\circ}$ radius around each point to guarantee a solution from PD routine. The PD vectors found are shown in the upper panels of Fig. 12. They follow the three conditions stated in Sect. 3, indicating the possible existence of a intrinsic spatial common motion.

For an initial determination of modulus and apex of both motions, a new grid of points evenly distributed along meridians and parallels is defined, so that apex and antapex are the new poles of the sphere. Once the PD is applied to the new points (Fig. 12, middle panels), we check that the vectors' moduli follow Herschel's formulation (lower panels Fig. 12). Initial results indicate the existence of a common motion, to which we assign a value for the Hercules stream of: apex $(l, b)=\left(237.5,-0^{\circ} 5\right), V=61.5 \mathrm{~km} \mathrm{~s}^{-1}$ and for Sirius stream: apex $(l, b)=\left(40,-6^{\circ}\right), V=18.01 \mathrm{~km} \mathrm{~s}^{-1}$, when taking, respectively, the values of $\mu=0.13^{\prime \prime} / \mathrm{yr}$ and $\mu=0.038^{\prime \prime} / \mathrm{yr}$ as the scaling factors for the sine functions. According to (F05), the Hercules stream has an apex $(l, b)=\left(230^{\circ} 8,-0^{\circ} 1\right)$ with $V=67.1 \mathrm{~km} \mathrm{~s}^{-1}$, and the Sirius stream has an apex $(l, b)=$ $\left(58^{\circ} 8,-34^{\circ} 1\right)$ with $V=9.6 \mathrm{~km} \mathrm{~s}^{-1}$.

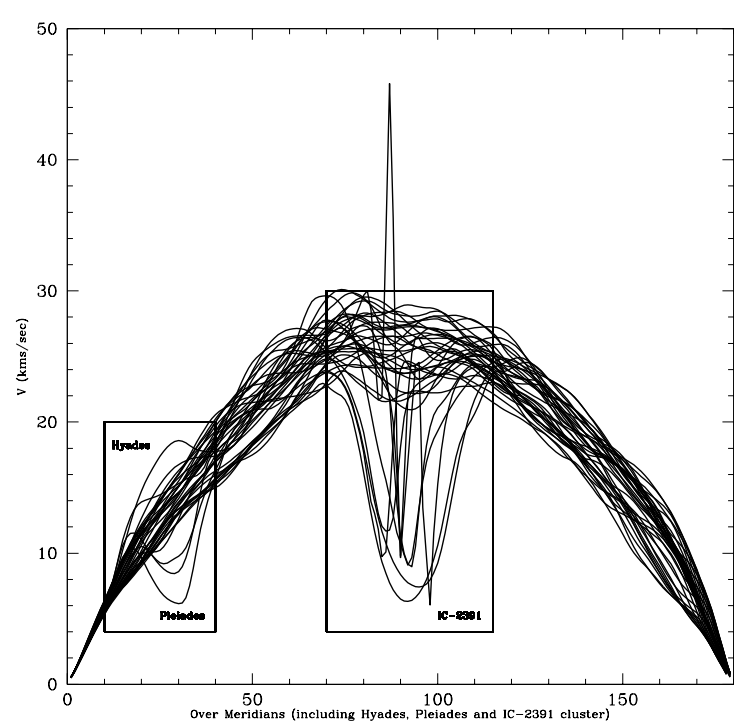

Fig. 10. Local deformations to Fig. 5a appear when the Hyades, IC-2391 and Pleiades clusters are included into Sample 1.

It is necessary to remark that both samples are contaminated, in our opinion, by stars that do not follow the stream's general trend. Nevertheless, it is not our aim to study them but rather to show that our method is able to detect the streams within a sample that certainly contains them. Our results are clear and stable, and they differ numerically from those cited by (F05), just because: (a) we correct the stellar proper motions for solar motion and (F05) does not, and (b) different methods are employed to measure the apex and the velocity.

We additionally made this same cross-checking, using the (A03) solar motion and the Mignard (2000) model of galactic rotation, and we again recover both streams. Differences between 5 and $10 \mathrm{~km} \mathrm{~s}^{-1}$ in $V$ appear, due to the quite different model of solar motion applied. Some smooth corrections also appear for the apex, but they continue near the galactic plane.

Returning to our data, the strength of the method is certainly proved, when the inclusion into the first preselected Sample 1, of data from known clusters with well defined motions, produces only smooth local but no general variations to the final results. Figure 10 shows the same kind of plot as Fig. 5a, when the Hyades, IC-2391 and Pleiades clusters are included. Similar deformations are introduced in Fig. 6a, when the Sirius supercluster is included as well.

\section{Discussion and conclusions}

In this paper we show a procedure that is able to detect trends of motion in samples of stars. Using proper motions and parallaxes, but not requiring radial velocities, we can obtain the modulus and direction of the motion. Therefore, it has the capability to detect stellar associations, moving groups and streams, particularly when working on extended areas.

Stellar streams have been known from many years. Between 1925 and 1930, Ralph Wilson and Harry Raymond with proper motions, and Gustav Strömberg with radial velocities, dealt with the two Kapteyn streams and other features in the velocity distribution. Streams were a main subject of 


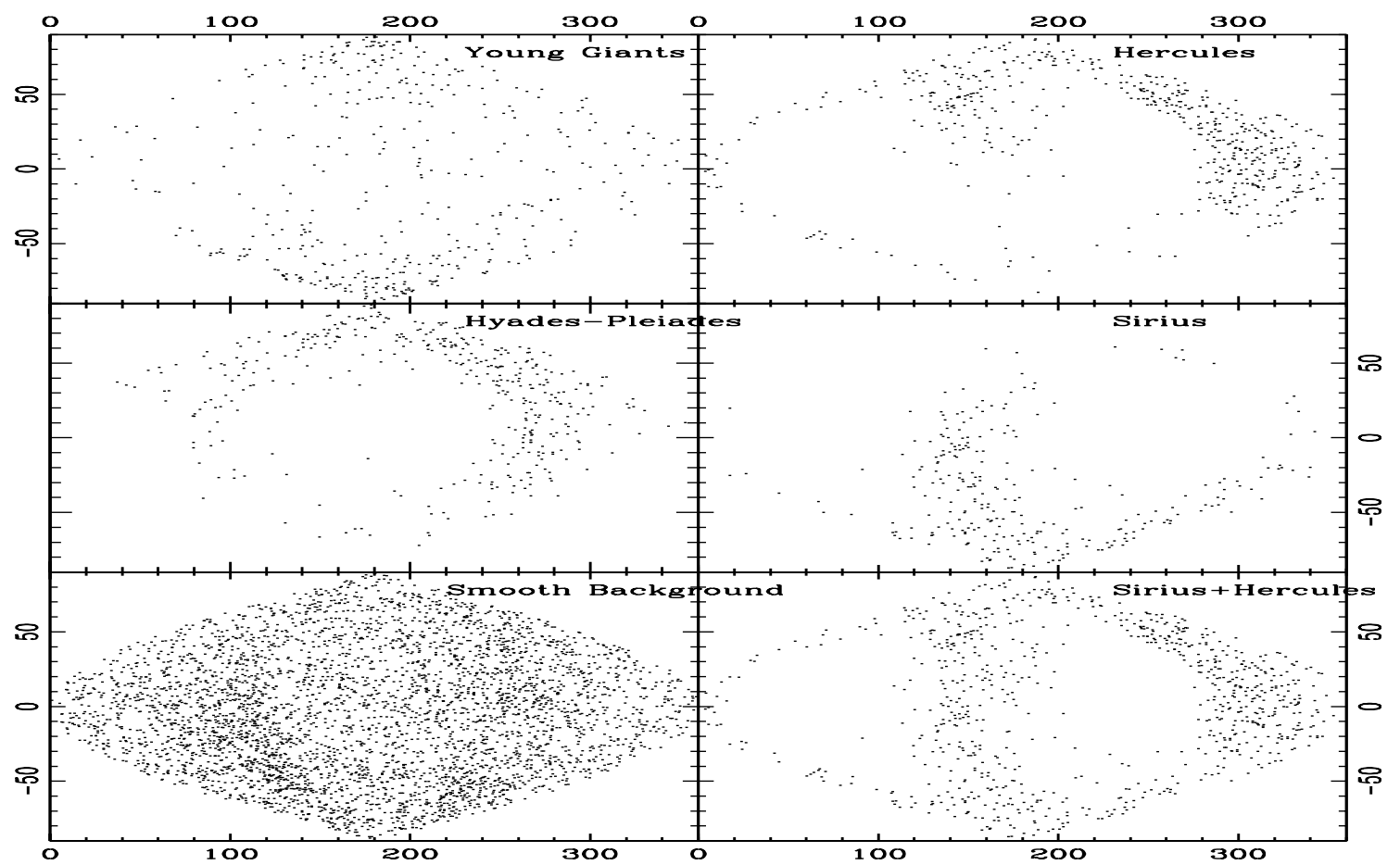

Fig. 11. Polar representation of the "dynamical streams" of Famaey et al. (2005), in galactic coordinates, already corrected for differential galactic rotation and solar motion.

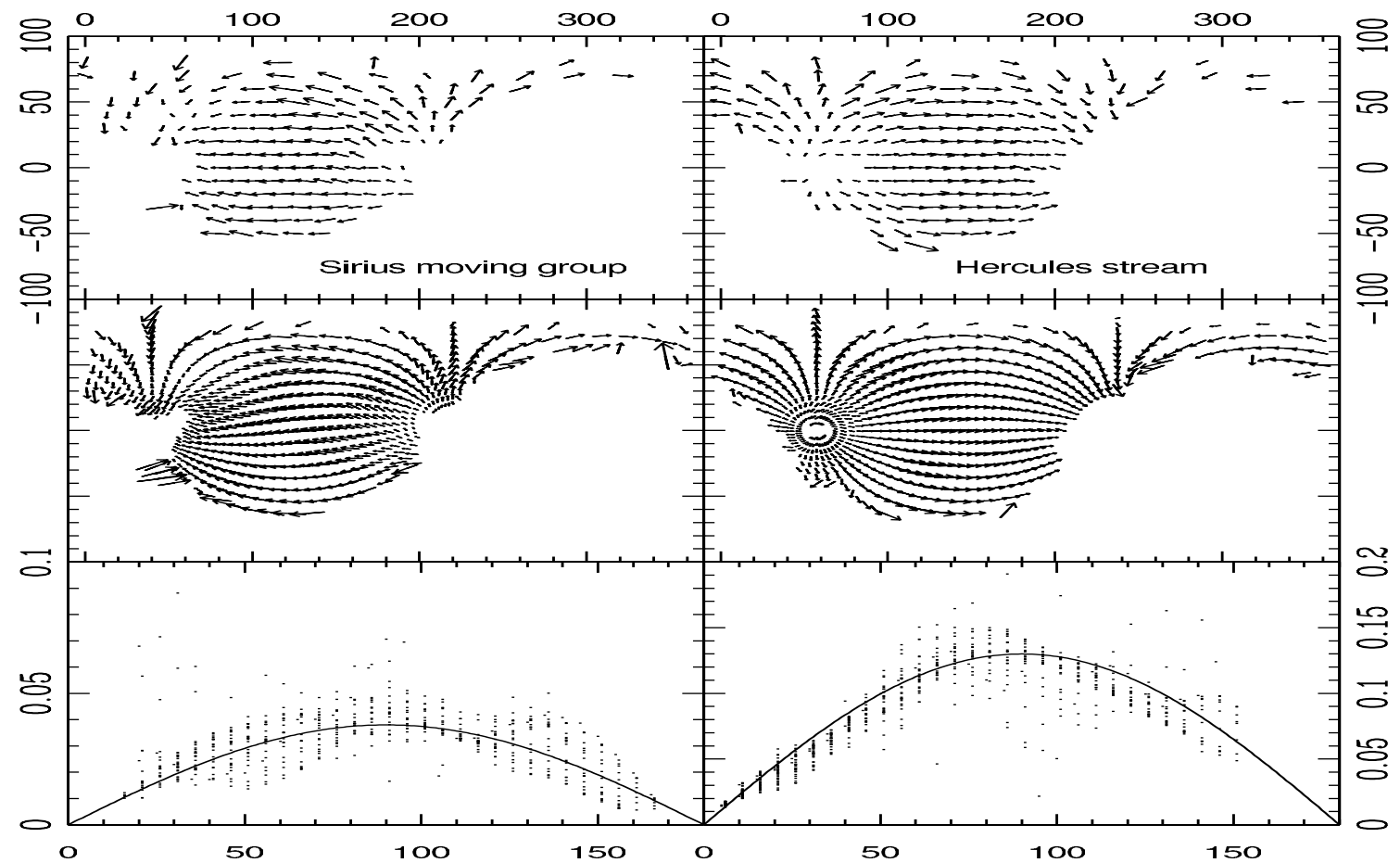

Fig. 12. Upper and middle panels: vectorial representation of the PD results, in galactic coordinates, for the Sirius and Hercules streams. Lower panels: PD vectors' moduli ("/yr) versus apex-position angle. A sine function is plotted with a solid line.

study by Eggen (1996a,b), who located some of them using $(U, V)$ velocities preferentially located in the second quadrant. Gozha (2001) made a study of Eggen's Groups, indicating that Stream I (associated with the Hyades cluster), and Stream II (Pleiades and Sirius Supercluster) are the most crowded ones.
The knowledge of distance, position, motion and the probability of membership for known stellar clusters, helps study the streams associated with them, but on the other hand, it makes the clusters an indispensable tool to begin with. The initial criterion we use to select stars as probable members of a common 


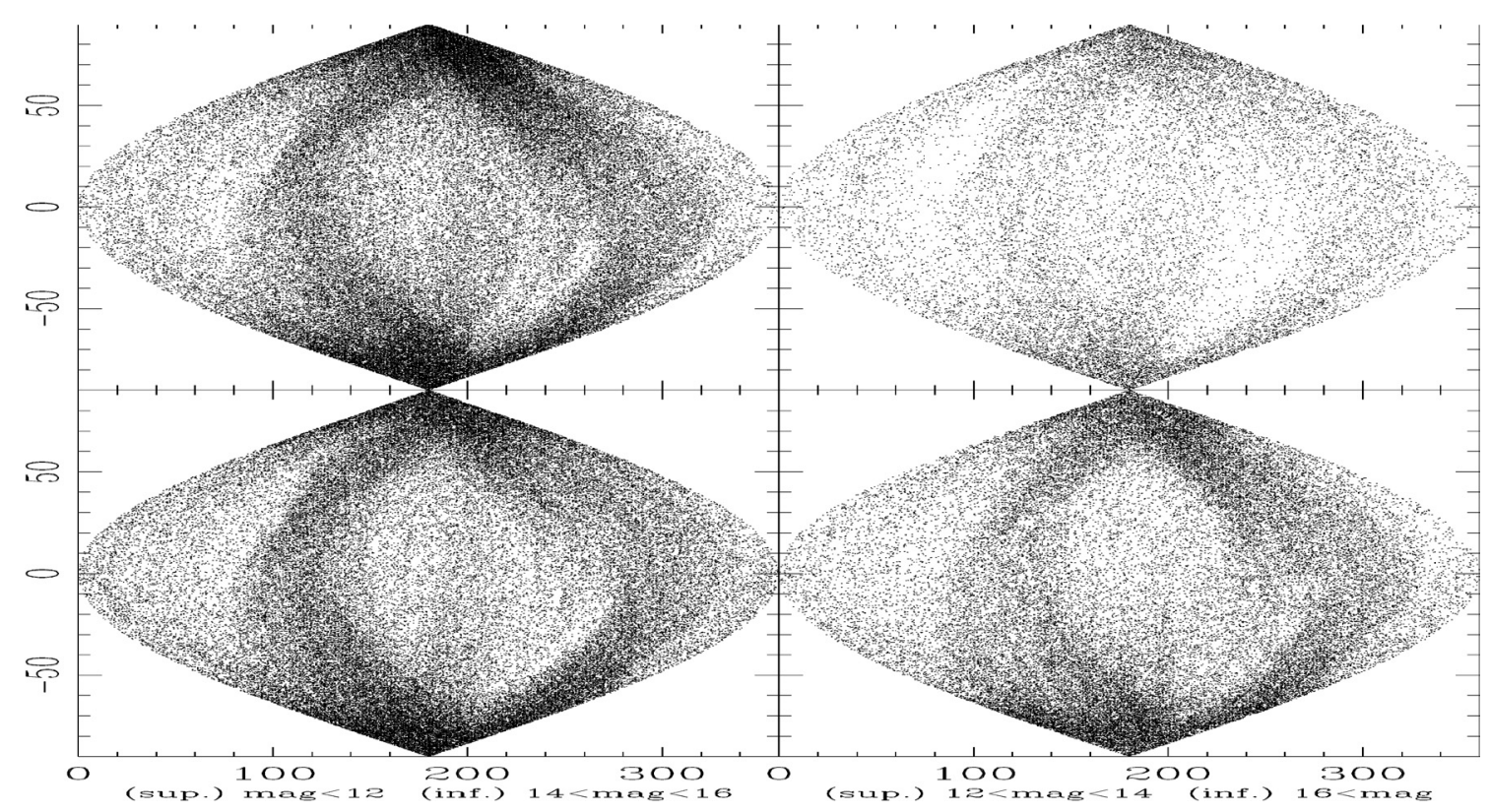

Fig. 13. Polar representation of the CPM for the NPM2 catalogue in galactic coordinates, separated by range of magnitude. It is possible to see a pattern similar to that found in Fig. 1 for the Hipparcos catalogue, which motivated this paper.

motion depends exclusively on the individual proper motion. We choose stars whose paths cross a preselected area, with no assumptions about space location or gravitational conditions (such as being linked to a known cluster). In this way it is possible to detect patterns of motion that make a portion, but not the total of the stellar motion. Certainly, this area is not chosen randomly, polar representation of CPM and the PD numerical routine help us in this selection.

As our method makes use of the parallaxes, this means that Hipparcos is the most extensive catalogue so far, to which it can be applied. Nevertheless, some additional evidence, supporting the fact that these patterns are extended, can be obtained from other databases, like the NPM2 catalogue (Hanson et al. 2003). This catalogue contains absolute proper motions for 232000 stars north of $\delta=-23^{\circ}$, in a blue apparent magnitude range from 8 to 18 . The catalogue covers $28 \%$ of the northern sky, lying near the plane of the Milky Way and many of the stars are included in fields away from the galactic plane. Figure 13 shows the equivalent to Fig. 1, using NPM2 original proper motions. The clear presence of an enhanced density zone of poles along a wide great circle, motivated this paper.

Detecting and measuring the different kinematic populations in the solar neighborhood is of the foremost importance. The structure of the local velocity distribution reveals information about the dynamic equilibrium of the solar neighborhood and the galaxy. Assuming the predominance of the global over the local galactic potential, the disturbing effect of a non-axisymmetric component, like the rotating bar, could explain some of the "large-scale" motions that many stars exhibit around us. On the other hand, local and temporal disturbances of the potential could also generate the observed groups of common velocity.

A precise cause for the patterns of motion detected in this paper is difficult to establish. For a discussion of the possible origins for such streams, refer to (F05). Further observations are required to accurately sample the solar neighborhood velocity field. On the other hand, pushing the distance limits outwards in the astrometric catalogues, makes it imperative to create suitable procedures in order to properly manage large amounts of data. Sampling a greatly extended volume of space, surely means that several other forces and motions come to play, and makes more difficult the detection of kinematically bound groups of stars.

The goal of this paper has been to develop a new way to study the kinematics of the solar neighborhood, in the search of streams, associations, clusters and any other space motion shared by a large number of stars, without being restricted by the availability of radial velocities.

Acknowledgements. We would like here to thank Dr. Jurgen Stock (1923-2004) for a full life of work and commitment to astrometry. This paper is dedicated to him, to honor all his achievements, the work he started will continue well into the future. We also deeply thank Bill van Altena (Yale University) for help with the English version of this paper.

\section{References}

Abad, C. 1996, Ph.D. Thesis, Publicaciones del Seminario Matemático García de Galdeano, Serie II, No. 52, Universidad de Zaragoza, Zaragoza, Spain

Abad, C., Vieira, K., Bongiovanni, A., Romero, L., \& Vicente, B. 2003, A\&A, 397, 345 (A03)

Agekyan, T. A., \& Popovich, G. A. 1993, AZh, 70, 122

Barbier-Brossat, M., \& Figon, P. 2000, A\&AS, 142, 217

Chereul, E., Creze, M., \& Bienayme, O. 1998, A\&A, 340, 384

De Simone, R. S., Wu, X., \& Treimane, S. 2004, MNRAS, 350, 627

Dehnen, W. 1998, AJ, 115, 2384

Dehnen, W. 2000, AJ, 119, 800

Dehnen, W., \& Binney, J. 1998, MNRAS, 298, 387

Eggen, O. J. 1996a, AJ, 111, 4, 1615 
Eggen, O. J. 1996b, AJ, 112, 4, 1595

Famaey, B., Jorissen, A., Luri, X., et al. 2005, A\&A, 430, 165 (F05)

Feast, M., \& Whitelock, P. 1997, MNRAS, 291, 683

Fux, R. 2001, A\&A, 373, 511

Gozha, M. L. 2001, Stellar Dynamics: From Classic to Modern, Proceedings of the International Conference held in Saint Petersberg, August 21-27, 2000, in honour of the 100th birthday of Professor K. F. Ogorodnikov (1900-1985), ed. L. P. Ossipkov, \& I. I. Nikiforov, Saint Petersburg: Sobolev Astronomical Institute, 60

Hanson, R. B., Klemola, A. R., Jones, B. F., \& Monet, D. G. 2004, AJ, 128,1430

Helmi, A., White, S. D. M., de Zeeuw, P. T., \& Zhao, H. 1999, Nature, 402,53

Jaschek, C., \& Valbousquet, A. 1992, A\&A, 255, 124

López-Corredoira, M., Hammersley, P. L., Garzón, F., et al. 2001, A\&A, 373, 139

Luyten, W. J. 1976, LHS Catalogue, University of Minnesota, Minneapolis, USA
Majewski, S. R., et al. 2003, Dark Matter in Galaxies, International Astronomical Union. Symposium No. 220, held 22-25 July, 2003 in Sydney, Australia

Makarov, V. V., \& Urban, S. 2000, MNRAS, 317, 289

Mihalas, D., \& Binney, J. 1981, Galactic Astronomy (San Francisco, USA: W. H. Freeman and Company)

Mignard, F. 2000, A\&A, 354, 522

Muhlbauer, G., \& Dehnen, W. 2003, A\&A, 401, 975

Perryman, M. A. C., \& ESA 1997, The HIPPARCOS and TYCHO catalogues. Astrometric and photometric star catalogues derived from the ESA HIPPARCOS Space Astrometry Mission (Noordwijk, Netherlands: ESA Publications Division)

Quillen, A. 2003, AJ, 125, 785

Stock, J., \& Abad, C. 1988, RMxAA, 16, 63

Schwan, H. 1991, A\&A, 243, 386

Sellwood, J. A., \& Binney, J. J. 2002, MNRAS, 336, 785

Trumpler, R. J., \& Weaver, H. F. 1953, Statistical Astronomy (Berkeley and Los Angeles, California, USA: University of California Press) 